\title{
Constraining the Emission Geometry and Mass of the White Dwarf Pulsar AR Sco Using the Rotating Vector Model
}

\author{
Louis du Plessis $^{1}$ (1) , Zorawar Wadiasingh ${ }^{1,2}$ (10), Christo Venter ${ }^{1}$ (10), and Alice K. Harding ${ }^{2}$ (1) \\ ${ }^{1}$ Centre for Space Research, North-West University, Private Bag X6001, Potchefstroom 2520, South Africa \\ ${ }^{2}$ Astrophysics Science Division, NASA Goddard Space Flight Center, Greenbelt, MD 20771, USA \\ Received 2019 July 11; revised 2019 October 9; accepted 2019 October 14; published 2019 December 10
}

\begin{abstract}
We apply the standard radio pulsar rotating vector model to the white dwarf (WD) pulsar AR Sco's optical polarization position angle swings folded at the WD's spin period as obtained by Buckley et al. Owing to the long duty cycle of spin pulsations with a good signal-to-noise ratio over the entire spin phase, in contrast to neutron star radio pulsars, we find well-constrained values for the magnetic obliquity $\alpha$ and observer viewing direction $\zeta$ with respect to the spin axis. We find $\cos \alpha=0.060_{-0.053}^{+0.050}$ and $\cos \zeta=0.49_{-0.08}^{+0.09}$, implying an orthogonal rotator with an observer angle $\zeta=60^{\circ} .4_{-6^{\circ} .0^{\circ}}$. This orthogonal nature of the rotator is consistent with the optical light curve consisting of two pulses per spin period, separated by $180^{\circ}$ in phase. Under the assumption that $\zeta \approx i$, where $i$ is the orbital inclination, and that the companion M star is Roche-lobe-filling, we obtain $m_{\mathrm{WD}}=1.00_{-0.10}^{+0.16} M_{\odot}$ for the WD mass. These polarization modeling results suggest the that nonthermal emission arises from a dipolar WD magnetosphere and close to the star, with synchrotron radiation (if nonzero pitch angles can be maintained) being the plausible loss mechanism, marking AR Sco as an exceptional system for future theoretical and observational study.

Unified Astronomy Thesaurus concepts: White dwarf stars (1799); Compact objects (288); Optical pulsars (1173); Rotation powered pulsars (1408); Non-thermal radiation sources (1119); Magnetic fields (994); Binary pulsars

(153); X-ray sources (1822)
\end{abstract}

\section{Introduction}

AR Scorpii (AR Sco) is an intriguing binary system containing a putative white dwarf (WD) with an M-dwarf companion. The system exhibits pulsed nonthermal radio, optical, and X-ray emission, likely of synchrotron radiation (SR) origin (Marsh et al. 2016; Buckley et al. 2017; Takata et al. 2018). AR Sco has a binary orbital period of $P_{\mathrm{b}}=3.56 \mathrm{hr}$, a WD spin period of $P=1.95$ minutes, and a beat period of 1.97 minutes. The light cylinder radius ${ }^{3}$ and orbital separation are $R_{\mathrm{LC}}=c / \Omega \sim 6 \times 10^{11} \mathrm{~cm}$ and $a \sim 8 \times$ $10^{10} \mathrm{~cm}$, respectively, with $\Omega$ being the spin angular frequency. Thus, the M star is located within the WD's magnetosphere at $a \approx 0.13 R_{\mathrm{LC}}$. A change in spin period $\dot{P}=3.9 \times 10^{-13} \mathrm{~s} \mathrm{~s}^{-1}$ was inferred by Marsh et al. (2016) but was disputed by Potter \& Buckley (2018a) who argued that the observations were too sparse to derive an accurate spin-down timescale for the WD pulsar. With more extensive observations, Stiller et al. (2018) firmly established $\dot{P}=7.18 \times 10^{-13} \mathrm{~s} \mathrm{~s}^{-1}$, which is almost twice as large as the value reported in Marsh et al. (2016), but consistent with the constraints in Potter \& Buckley (2018a). The concomitant spin-down power is $\dot{E}_{\text {rot }}=I_{\mathrm{WD}} \Omega \dot{\Omega} \approx 5 \times$ $10^{33} \mathrm{erg} \mathrm{s}^{-1}$ for a fiducial WD moment of inertia $I_{\mathrm{WD}}=3 \times$ $10^{50} \mathrm{~g} \mathrm{~cm}^{2}$. A lack of Doppler-broadened emission lines from accreting gas suggests the absence of an accretion disk. ${ }^{4}$ More recently, X-ray data have established no evidence of an accretion column, and spectral analysis of the subdominant pulsed component suggests it is nonthermal (Takata et al. 2018). These

\footnotetext{
3 This is the radius where the corotation speed equals that of light in vacuum. 4 This is supported by the fact that the X-ray luminosity is only $4 \%$ of the total, optically dominated observed luminosity and is only $\sim 1 \%$ of the X-ray luminosities of typical intermediate polars, and also from the fact that all optical and ultraviolet emission lines originate from the irradiated face of the M-dwarf companion.
}

two characteristics of observed spin-down and the absence of an accretion disk led Buckley et al. (2017) to attribute the observed nonthermal luminosity to magnetic dipole radiation from the WD, conclusively establishing AR Sco as the first known WD pulsar, analogous to rotation-powered neutron star pulsars.

The WD is highly magnetized (with a polar surface field of $B_{\mathrm{p}} \sim 8 \times 10^{8} \mathrm{G}$ estimated by setting $\dot{E}_{\text {rot }}=\dot{E}_{\text {md }}$, with $\dot{E}_{\text {md }}$ being the energy loss due to a magnetic dipole rotating in vacuum) and its optical emission is strongly linearly polarized (up to 40\%; Buckley et al. 2017). Potter \& Buckley (2018b) conducted extensive follow-up optical observations on AR Sco, reporting that the linear flux, circular flux, and position angle are coupled to the spin period $P$ of the WD. The polarization position angle (PPA; $\psi$ ) versus time indicates clear periodic emission. In this paper, we model the linear polarization signature with the rotating vector model (RVM; Radhakrishnan $\&$ Cooke 1969):

$$
\tan \left(\psi-\psi_{0}\right)=\frac{\sin \alpha \sin \left(\phi-\phi_{0}\right)}{\sin \zeta \cos \alpha-\cos \zeta \sin \alpha \cos \left(\phi-\phi_{0}\right)},
$$

with $\alpha$ being the magnetic inclination angle of the magnetic dipole moment $\boldsymbol{\mu}$ and $\zeta$ being the observer angle (observer's line of sight), both measured with respect to the WD's rotation axis $\Omega, \phi$ the rotational phase (WD spin), and the parameters $\phi_{0}$ and $\psi_{0}$ are used to define a fiducial plane. The light curve from Buckley et al. (2017) manifests double peaks with a more intense first peak followed by a dimmer second peak, exhibiting a peak separation of $\sim 180^{\circ}$. In addition, the PPA makes a $180^{\circ}$ sweep. These facts imply that the WD may be an orthogonal rotator if the emission originates close to its polar caps (Geng et al. 2016; Buckley et al. 2017). We show that our solution for $\alpha$ using the RVM supports this conjecture. 
The observations by Marsh et al. (2016) indicate irradiation of the side of the WD facing the companion. This forms part of the observed sinusoidal radial velocity profile, suggesting that the two stars are tidally locked (Buckley et al. 2017; Takata et al. 2017; Potter \& Buckley 2018b). To account for the observed nonthermal radiation, models invoke injection of relativistic electrons by the companion along the magnetic field lines of the WD, where they are trapped and accelerated (Geng et al. 2016; Buckley et al. 2017; Takata et al. 2017). However, different proposed scenarios place the nonthermal emission regions in different spatial locales. Geng et al. (2016) noted that the Goldreich-Julian charge number density (Goldreich \& Julian 1969) of the WD is much lower than required by the observed SR spectrum and thus argued that the emission should originate closer to the companion (they suggest an intrabinary shock caused by the interacting stellar winds; Geng et al. 2016; Marsh et al. 2016) where the particle number density is higher. They noted that the spin-down luminosity of the WD is sufficient to power the emission of the system. On the other hand, upon an injection of relativistic particles from the companion, emission may originate from near the magnetic poles of the WD by means of pulsar emission mechanisms (with the emission from downwardmoving particles being directed toward the WD; see Buckley et al. 2017; Takata et al. 2017; Potter \& Buckley 2018b; Takata \& Cheng 2019). This is supported by the geometric model of Potter \& Buckley (2018b) that can reproduce the observed polarization signatures if the emission location is taken to be at the magnetic poles of the WD. In what follows, we demonstrate that the $\mathrm{RVM}^{5}$ provides an excellent fit to the PPA curve, thereby favoring the magnetospheric scenario, if particles sustain small pitch angles. However, we note that this hypothesis may not be unique, since Takata \& Cheng (2019) claim that they can also reproduce the polarization properties using an independent emission model in which the particle pitch angles evolve with time and become quite large at the emission regions, which are incidentally also located relatively close to the WD polar caps.

The structure of this article is as follows. In Section 2 we discuss the folding of the data, code calibration, and fits. We present our results in Sections 3. Our discussion and conclusions follow in Sections 4 and 5.

\section{Method}

\subsection{Folding of Data}

We use the PPA data from Buckley et al. (2017), comprising observations on 2016 March 14 in the 340-900 nm range. We obtain the minimum PPA of the data set and define the corresponding time $t_{0}$ as the starting point to fold the data set. A few data points $(\sim 3 \%)$ deviate from the average PPA versus $\phi$ curve, but we note that the folding is affected by the choice of $t_{0}$. We remedy this convention issue by generating smoothed PPA curves with a kernel density estimation and a Gaussian kernel technique. By inspecting the deviation between the smoothed curve and the folded data, we assign a new convention to the points with a large deviation by shifting

\footnotetext{
5 It is important to note that the RVM is a purely geometrical model, and as such cannot make any statements about light-curve shapes or spectra expected from AR Sco. We cannot constrain particle energies, injection rates, or the acceleration process within this framework. We defer the construction of a full emission model to a future paper.
}

these points by $180^{\circ}$ (which is the intrinsic uncertainty of the convention assigned to the PPA, i.e., there is an ambiguity in the parallel and antiparallel directions). We then bin the data into 30 rotational phase bins. The predicted values of $\psi$ from Equation (1) are discontinuous, since the arctangent function is discontinuous at $\phi_{\text {disc }}=\arccos (\tan \zeta / \tan \alpha)$. Using this expression, we locate the discontinuities and shift the predicted PPA by $360^{\circ}$ at these phases to finally obtain a smooth, continuous PPA model.

\subsection{Code Verification and Best Fit}

We adopt the convention of Everett \& Weisberg (2001), letting $\psi$ increase in the counterclockwise direction. Thus we define $\psi^{\prime}=-\psi$. A Bayesian likelihood approach is employed to constrain the RVM using the optical PPA data. We define our "best fit" as the 50th percentile or median in the posterior distribution of the model parameters. We verified our code by obtaining and comparing independent RVM fits of radio pulsar data (Everett \& Weisberg 2001), yielding consistent $\alpha$ and $\zeta$ values, within uncertainties.

For the Bayesian analysis, we employ a Markov Chain Monte Carlo technique (Foreman-Mackey et al. 2013) with 50 walkers, 40,000 steps, and a step burn-in of 14,000. We maximize the following likelihood function:

$$
\begin{aligned}
& \ln p\left(y \mid \phi, \alpha, \beta, \phi_{0}, \psi_{0}, f\right) \\
& =-0.5 \Sigma_{n}\left[\frac{\left(y_{n}-H\left(\phi, \alpha, \beta, \phi_{0}, \psi_{0}\right)\right)^{2}}{S_{n}^{2}}+\ln \left(2 \pi S_{n}^{2}\right)\right],
\end{aligned}
$$

where $S_{n}^{2}=\sigma_{n}^{2}+f^{2}\left(H\left(\phi, \alpha, \beta, \phi_{0}, \psi_{0}\right)\right)^{2}, y$ represents the data values, $\sigma$ represents the uncertainties of the data (assumed to follow a Gaussian distribution), and $H$ represents the model values. The factor $f$ compensates for the case when the PPA uncertainties are underestimated. We assume uniform priors on the cosine of $\alpha$ and $\zeta$ (rather than the angles themselves). For $\cos \zeta$, this is an appropriate choice from Copernican arguments. For $\cos \alpha$, this convention is less justified, but nevertheless ultimately immaterial for the present context; we have verified there is no dramatic change in resulting uncertainties with a uniform prior choice on $\alpha$ rather than $\cos \alpha$.

\section{Results}

\subsection{Constraints on WD Geometry}

The red curve in Figure 1 depicts the best-fit $\mathrm{RVM}^{6}$ to the polarization data, with yellow curves associated with a random selection of parameters from the posterior distribution of $\cos \alpha$ and $\cos \zeta$. The uncertainties for the best fit are taken to be the $68 \%$ probability around the median, i.e., the 16 th and 84 th percentile values. From our best fits of these quantities, we obtain $\alpha=86^{\circ} 6_{-2}^{+3.0} .0$ and $\zeta=60^{\circ} .4_{-6}^{+5^{\circ} .3}$. For the uncertainty parameter $\ln (f)$, only the maximum of $\ln (f)$ is constrained, and $f$ is quite small, thus we conclude the data are described well by the model without the inclusion of this term.

The correlation or degeneracy seen between $\phi_{0}$ and $\psi_{0}$ in Figure 2 owes to the fact that these parameters translate the model horizontally and vertically; thus, a natural degeneracy exists, since the model is cyclic and a similar fit may be

\footnotetext{
6 See the Appendix for a parameter study that indicates the behavior of the RVM for different choices of $\alpha$ and $\zeta$.
} 
obtained for different choices of $\phi_{0}$ and $\psi_{0}$. This is also the reason why small, disconnected contours could be eliminated from Figure 2 by constraining the priors of the nuisance parameters $\phi_{0}$ and $\psi_{0}$. The large duty cycle leads to relatively small uncertainties on both $\alpha$ and $\zeta$ as compared to the case of known radio pulsars. The pulses of the latter typically have small duty cycles and thus relatively large uncertainties on $\alpha$ (the impact angle $\beta=\zeta-\alpha$ is typically better constrained, given visibility requirements, but $\zeta$ may remain illconstrained).

\subsection{Constraints on WD Mass}

Marsh et al. (2016) reported the mass ratio of AR Sco as $1 / q=M_{\mathrm{M}} / M_{\mathrm{WD}} \geqslant 0.35$, assuming $M_{\mathrm{WD}}=0.8 M_{\odot}$ and $M_{\mathrm{M}}=0.3 M_{\odot}$ as a natural pairing for the system that is located at a distance of $d=116 \pm 16 \mathrm{pc}$. They also measured the radial velocity of the M star, $K_{2}=295 \pm 4 \mathrm{k} \mathrm{s}^{-1}$, and obtained the following mass function

$$
m_{\mathrm{WD}} \sin ^{3} i\left(\frac{q}{1+q}\right)^{2}=\frac{P_{\mathrm{b}} K_{2}^{3}}{2 \pi G} \approx 0.395_{-0.0158}^{+0.0163} M_{\odot} .
$$

They report no significant evidence of nonsinusoidal radial velocities, thus suggesting little overestimation of the true amplitude owing to irradiation. Based on the M star's emission line velocities, Marsh et al. (2016) infer $q \lesssim 2.86$, perhaps close to the cited maximum $(q \sim 2.8)$ under the assumption that the M star is close to filling its Roche lobe. Moreover, from spectroscopic comparison to model atmospheres they conclude that the radius of the $\mathrm{M}$ star $R_{\mathrm{M}} \approx 0.36 R_{\odot}$ using a mass ${ }^{7}$ of $m_{\mathrm{M}} \approx 0.29 M_{\odot}$ via the volume-equivalent Roche radius approximation of Paczyński (1971),

$$
\frac{1}{3}\left(\frac{2 G m_{\mathrm{M}} P_{\mathrm{b}}^{2}}{3 \pi^{2}}\right)^{1 / 3} \approx R_{\mathrm{M}} \approx 0.36 R_{\odot} .
$$

Finally, based on the stellar radius estimate and the stellar brightness, they quote a mass estimate of $m_{\mathrm{M}} \approx$ $0.3(d / 116 \mathrm{pc})^{3} M_{\odot}$. A more accurate parallax distance of $d=117.8 \pm 0.6 \mathrm{pc}$ was reported by Stiller et al. (2018), implying $m_{\mathrm{M}} \approx 0.31 M_{\odot}$ when inverting the above massdistance relation, suggesting general consistency with the estimate $m_{\mathrm{M}} \sim 0.3 M_{\odot}$. Substitution of $m_{\mathrm{M}} \sim 0.3 M_{\odot}$ into Equation (3) and demanding that $m_{\mathrm{WD}}$ is below the Chandrasekhar mass limit of $1.44 M_{\odot}$ yields the constraint $i \gtrsim 47^{\circ}$.

The mass $m_{\mathrm{WD}}$ is generally ${ }^{8}$ insensitive to the value of $m_{\mathrm{M}}$, as is readily apparent from the $q \gg 1$ limit of Equation (3), and therefore also to any systematic errors associated with observational determinations of $R_{\mathrm{M}}$ and also the approximation employed in Equation (4). Constraints on $m_{\mathrm{WD}}$ may be obtained under the assumption $\zeta \sim i$, which is generally expected from formation/evolution and observed in other contexts (e.g., Albrecht et al. 2007; Watson et al. 2011). Note

\footnotetext{
7 This choice of mass is due to the M5 spectral type of the M-dwarf and is also typical of donor-star masses in other systems having similar orbital periods as AR Sco.

8 This is true as long as $q$ is in fact $\gg 1$. If $q \sim 1$ then $m_{\mathrm{WD}}$ is sensitive on the value of $q$. For our assumed value of $q \sim 3$, there is a small influence of $m_{\mathrm{WD}}$
}

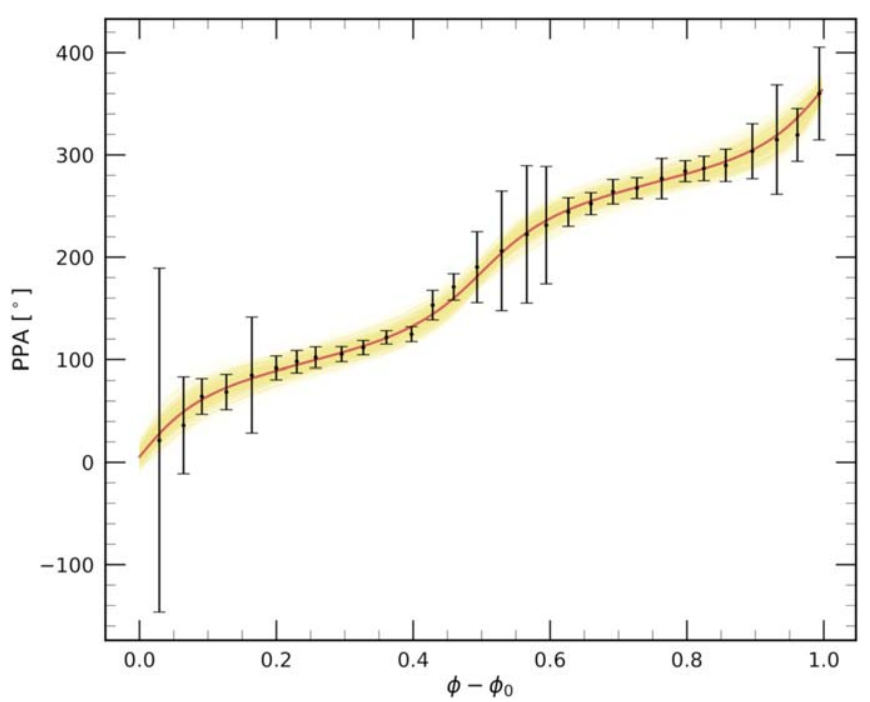

Figure 1. The best-fit RVM solution (red line) for the PPA data using an MCMC technique with the likelihood parameter $f$ included, also showing ensemble plots (possible fits) as yellow lines. The abscissa is chosen such that the observer crosses the WD fiducial plane, defined by the magnetic moment and spin vectors, at phase zero.

that the spin angular momentum of the WD is about two orders of magnitude inferior to the total orbital angular momentum. Since it is likely that the WD was spun up to its exceptionally low period of $P=117 \mathrm{~s}$ via past accretion episodes, the transfer of angular momentum would tend to align the WD spin to the orbit angular momentum. Allowing $m_{\mathrm{M}}$ be a free parameter, while propagating normally distributed uncertainties for $K_{2}$ and $\cos \zeta$ (see Figure 2) in Equation (3) yields a band of allowable values of $\left\{m_{\mathrm{M}}, m_{\mathrm{WD}}\right\}$ depicted in Figure 3. Adopting $R_{\mathrm{M}} \approx$ $0.36 R_{\odot}$ and solving the system of equations Equations (3) and (4) yields in $m_{\mathrm{WD}}=1.00_{-0.10}^{+0.16} M_{\odot}$ for the median, with uncertainties for the $68 \%$ containment region of probability. This yields $q=3.45_{-0.45}^{+0.66}$ that is somewhat in tension with Marsh et al.'s (2016) quoted $q \lesssim 2.86$ derived from the velocity amplitudes of atomic emission lines relative to the M, but not meaningfully so, given the allowable range of uncertainties and systematics which may be present in the M star mass/radius estimate.

\subsection{Geodetic Precession}

If we allow misalignment of the spin and orbital axes $\zeta \neq i$, this may explain why the optical maximum does not occur at inferior conjunction of the WD (Buckley et al. 2017). Katz (2017) proposed that this mismatch may be explained either by dissipation in a bow wave or by misalignment between the WD spin axis and both the orbital axis and the WD's oblique magnetic moment as well as potential oblateness of the WD, causing precession of the spin axis. The latter would lead to variable heating of the companion surface along with a drift in the phase of the optical maximum, with a period of decades. Peterson et al. (2019) analyzed a century's worth of optical photometry on AR Sco but could not detect any precessional period as suggested by Katz (2017), although this period may be much longer for a larger angle of misalignment between the orbital plane and the WD spin axis, or a smaller oblateness. 


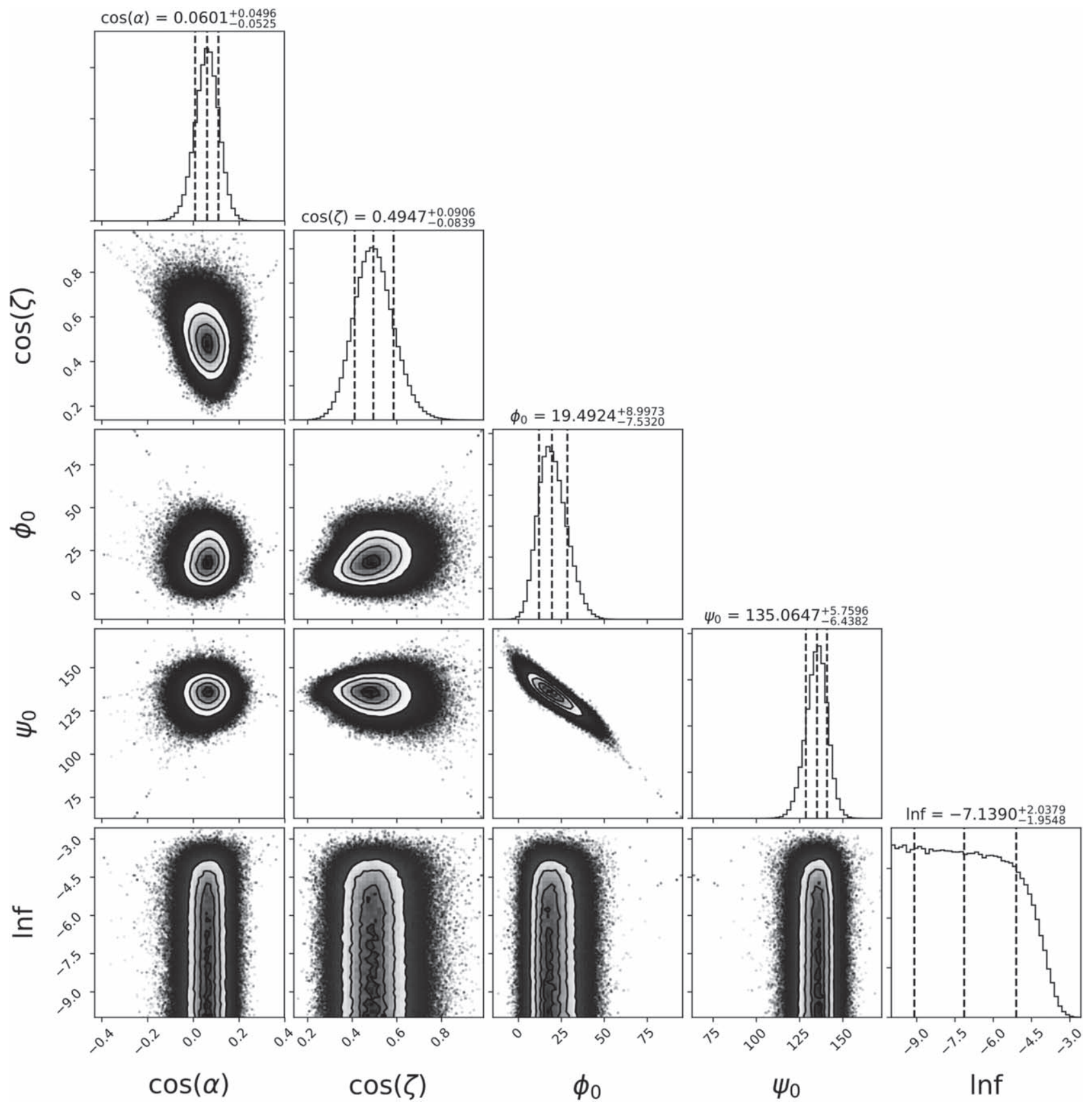

Figure 2. The best-fit for the model implementing $\cos \alpha, \cos \zeta$, and a likelihood parameter $f$. We found $\cos \alpha=0.060_{-0.053}^{+0.050}, \cos \zeta=0.49_{-0.08}^{+0.09}, \phi_{0}=\left(19^{\circ} .5_{-8.4}^{+9^{\circ}}{ }^{1}\right)$, $\psi_{0}=\left(135^{\circ} 1_{-6.4}^{+5.8}\right)$, and $\ln f=-7.1_{-2.0}^{+2.0}$. The $\phi_{0}$ and $\psi_{0}$ are nuisance parameters.

We suggest that effective spin-orbit interaction may result in the precession of the WD pulsar spin axis (Damour \& Ruffini 1974; Esposito \& Harrison 1975) owing to the metric curvature of the companion, even if the WD is not oblate. While any precession in the system may be dominated by electromagnetic influences of the companion, the general relativity (GR) rate calculated below is the minimum rate in absence of any electromagnetic torques. In the framework of classical GR, the angular geodetic precession rate $\omega_{p}$ is derived by Barker \& O'Connell (1975a, 1975b). The rate of precession is insensitive to $m_{\mathrm{WD}}$ and may be estimated independent of the assumption $\zeta \approx i$,

$$
\begin{aligned}
\omega_{p} & =\frac{G^{2 / 3}}{2 c^{2}\left(1-\varepsilon^{2}\right)}\left(\frac{2 \pi}{P_{\mathrm{b}}}\right)^{5 / 3} m_{\mathrm{M}}^{2 / 3} \frac{3+4 q}{(1+q)^{4 / 3}} \\
& \sim \frac{4 \times 10^{-10} \mathrm{rad} \mathrm{s}^{-1}}{1-\varepsilon^{2}} \approx \frac{0.8 \mathrm{deg} \mathrm{yr}^{-1}}{1-\varepsilon^{2}} .
\end{aligned}
$$

Since the orbital eccentricity $\varepsilon$ is presumably close to zero, the numerical value in Equation (5) constitutes a lower limit to the expected precession rate. This rate is similar to pulsar systems where such precession has been detected (e.g., B1913+16 


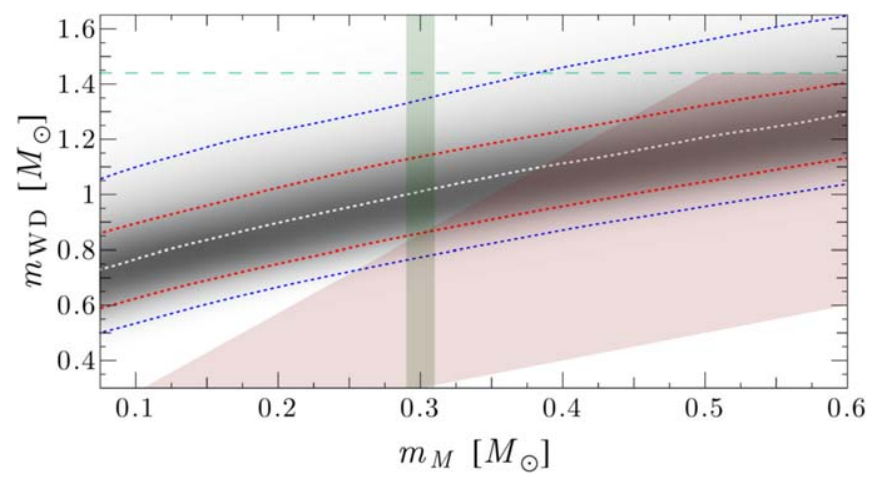

Figure 3. Various constraints on the the component masses $\left\{m_{\mathrm{M}}, m_{\mathrm{WD}}\right\}$. The gray band depicts the probability density in the mass function Equation (3) with the assumption $\zeta \approx i$ constructed by propagating uncertainties from the fitted $\cos \zeta$ in this paper and uncertainties of the radial velocity in Marsh et al. (2016). The white dashed curve is the median of the probability distribution of $m_{\mathrm{WD}}$ while $\{$ red, blue $\}$ curves delineate regions of $\{68 \%, 95 \%\}$ probability. The pink band is the constraint $1<q<2.86$ from Marsh et al. (2016), while the green band portrays $m_{\mathrm{M}} \sim 0.3 M_{\odot}$ as inferred from Equation (4). The Chandrasekhar limit of $m_{\mathrm{WD}}<1.44 M_{\odot}$ is traced by the cyan dashed line.

Weisberg et al. 1989; Cordes et al. 1990; Kramer 1998) over decade timescales. The observable scope of precession depends on the degree of misalignment; if $\zeta$ is significantly different from $i$, such precession (including that by oblateness of the WD) may be imprinted on the pulses and PPA swings of AR Sco and therefore changes in $\zeta$ values may be detectable on similar decade timescales as in pulsar binary systems. That is, from long-term time evolution of pulses and polarization data, the degree of misalignment may be estimated. Moreover, if well-characterized, such precession also affords an independent constraint on the component masses.

Takata \& Cheng (2019) also note that a detailed comparison between model and measured PPA sweeps may constrain the orientation of the spin axis of the WD. In the future, we will study polarization data as a function of orbital phase $\phi_{\mathrm{b}}$ to constrain the effects of precession that a varying $\alpha$ or $\zeta$ versus $\phi_{\mathrm{b}}$ may point to. In addition, predictions from a full emission model should lead to predictions of the PPA evolution that may be fit to data to constrain the WD spin axis alignment with the orbital axis and/or precession in the system. It is hoped that contraints on precession may teach us more about the system's characteristics, analogous to the case of PSR J1906+0746 where observations over several years of this precessing pulsar revealed the average structure of the radio beam (Desvignes et al. 2019).

\section{Discussion}

\subsection{Assumptions Regarding the Particle Pitch Angle}

Using our fits for $\alpha$ and $\zeta$, we can constrain the geometry of the emission region for the optical radiation. The derivation of the RVM (see Appendix of du Plessis et al. 2019) assumes that the observer samples emission that is tangent to local magnetic field lines and that the polarization vector is in the poloidal plane. This is a good approximation in either SR or curvature radiation $(\mathrm{CR})$ scenarios, provided that the particle momentum parallel to the field is relativistic and the relativistic particles have small pitch angles.

At first sight, this assumption of small pitch angle $\eta$ seems plausible since, for a large Lorentz factor $\gamma$, the pitch angle is $\eta \sim \theta_{\gamma} \sim 1 / \gamma$ (see Equation (10) where we estimate that the particles radiating optical emission have $\gamma \sim 30-100$ from the spectrum, depending on $B$ and $\eta$, and that $\eta \sim 10^{-4}$ if $\gamma \sim 5$ ). However, both $\gamma$ and $\eta$ evolve with distance as the particles move along the $B$-field lines. For example, Takata et al. (2017) solve an approximate form ${ }^{9}$ of the coupled set of equations that describe the evolution of $\gamma$ and perpendicular momentum $p_{\perp}$, used earlier by Harding et al. (2005) in the context of neutron star pulsars. Takata et al. $(2017,2018)$ and Takata \& Cheng (2019) assume that relativistic particles are injected from the companion and travel into the WD magnetosphere (along closed $B$-field lines, an assumption based on $\alpha \sim 60^{\circ}<90^{\circ}$ ) before radiating significantly, since the SR timescale is much longer than the light-crossing timescale $r / c$ at the companion position $(r \sim a)$. They then study a magnetic mirror effect in which the first adiabatic invariant $\mu \propto p_{\perp}^{2} / B$ is conserved and find that if the initial pitch angle is large enough ( $\sin \eta_{0}>0.05$, i.e., outside a loss cone set by initial conditions), the mirror effect operates and the pitch angles increase to $\sim \pi / 2$ at the mirror point (thus violating the RVM assumption of tangential emission) as the particles move closer to the WD surface. In this case, significant emission occurs at the mirror point, since SR losses increase rapidly with $B$ and $\eta$, before the particles return outward.

One can solve for the emission height at which the SR loss timescale equals the light-crossing timescale $r / c$ :

$$
\frac{r}{a} \approx 0.21 \gamma_{50}^{1 / 5} \mu_{35}^{2 / 5} \eta_{0.1}^{2 / 5} \sim 0.03 R_{\mathrm{LC}}
$$

where $\gamma_{50}=\gamma / 50, \mu_{35}=\mu / 10^{35} \mathrm{G} \mathrm{cm}^{3}, \mu=0.5 B_{\mathrm{s}} R_{\mathrm{WD}}^{3}$ is the magnetic moment, and $\eta_{0.1}=\eta / 0.1$ is the pitch angle. According to the calculations of Takata et al. (2017), the mirror effect operates and the particles turn around at $r \sim 0.25 a$, for $\gamma=50, \mu=6.5 \times 10^{34} \mathrm{G} \mathrm{cm}^{3}$, and $\eta=0.1$. However, if we choose plausible values of $\gamma=150$ and $\mu=2 \times 10^{35} \mathrm{G} \mathrm{cm}^{3}$ (which is closer to that implied by the estimated surface magnetic field $B_{\mathrm{s}}$, see Equation (7)), then Equation (6) implies that $r \sim 0.34 a>0.25 a$. This suggests that $p_{\perp}^{2} / B$ is no longer an invariant and the mirror effect will not operate, but the particles will lose all their energy abruptly suffering catastrophic SR losses. Thus, the pitch angle may never attain very large values.

We thus note that small pitch angles (as assumed by the RVM) are plausible in two cases: (i) when some parameter choices exist where $t_{\mathrm{SR}}>t_{\text {cross }}$ at a height $r$ that is a substantial fraction of $a$, so that the particles radiate all their energy before undergoing magnetic mirroring; (ii) when particles with small enough initial pitch angles (e.g., $\sin \eta_{0} \lesssim 0.05$ ) fall in the loss cone and are not impacted by the magnetic mirror. A complete model, solving the particle dynamics generally (for any $\eta$ and $\gamma$, and not in the drift approximation) to predict the light curves, spectrum, and polarization properties of AR Sco will be able to address this issue more fully.

\footnotetext{
9 The set of equations used by Takata et al. (2017) are valid only for $\gamma \gg 1$ and $\eta \ll 1$, and assumes that the accelerating $E$-field is screened. The more general form of the equations used by Harding et al. (2005) includes a term involving a nonzero $E$-field, but are subject to the drift approximation, where the motion of the guiding center is considered and the helical motion is averaged over gyrophase. We concur with Takata et al. (2017) that the local accelerating $E$-field (parallel to the local $B$-field) is probably screened-see Equation (16) below. We do not address the details of the acceleration process in this paper, other than to note it seems sufficient to accelerate particles to very large $\gamma_{\mathrm{e}, \max } \sim e B R_{\text {comp }} / m_{\mathrm{e}} c^{2} \sim 10^{6}-10^{8}$. Such high factors are needed if SR is to account for the observed X-ray emission, as noted in Equation (13).
} 


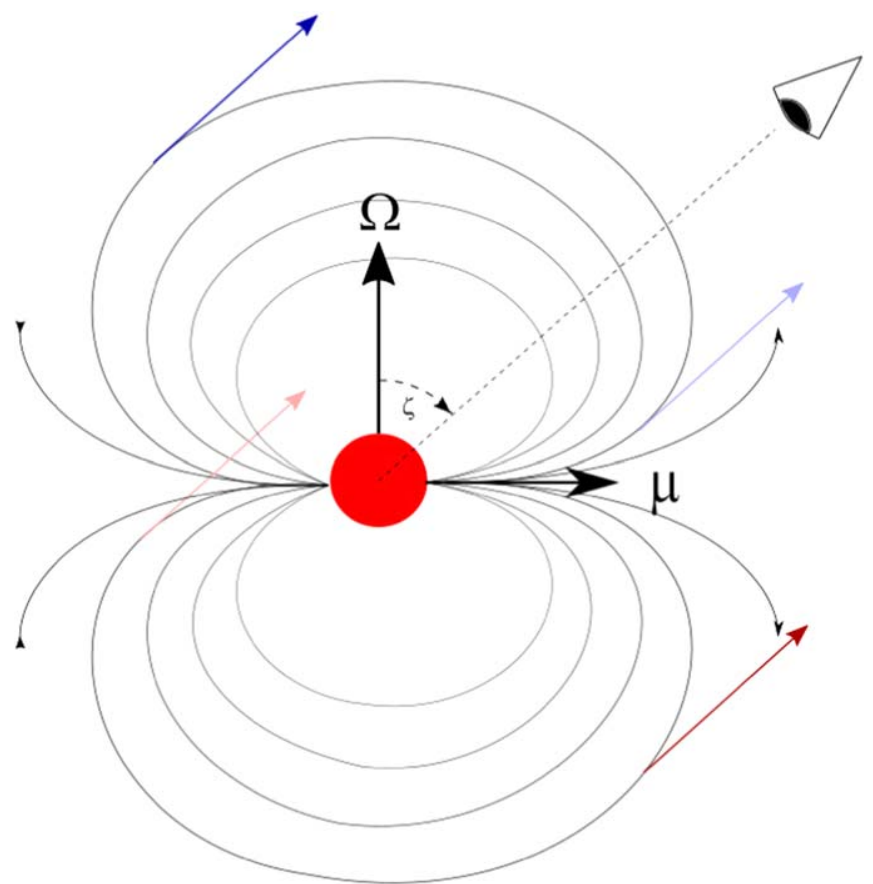

Figure 4. Schematic diagram indicating an orthogonal rotator with a dipolar field, as well as four possible solutions for emission that is radiated tangent to the local magnetic field lines and is pointing toward a distant observer. The spin axis is indicated by $\Omega$, the magnetic moment by $\boldsymbol{\mu}$, and the observer angle by $\zeta$. The red arrows indicate inflowing while the blue arrows indicate outflowing particles, as defined with respect to the nearest magnetic pole.

\subsection{Constraints on the Emission Geometry}

In a dipolar magnetosphere, multiple locations satisfy the viewing constraints derivable using our $\alpha$ and $\zeta$ fits within the framework of the RVM, which assumes emission to be tangential to the local $B$-field, irrespective of altitude, since a dipolar field is self-similar (additional spectral information may help constrain the actual emission heights, not just the emission directions). Depending on the current system, any nonzero subset of these locations could participate to produce the observed PPA curve. Thus, since the RVM variation is seen over the entire spin rotation of the WD, at least one of the multiple solutions must be realized (e.g., the model Potter \& Buckley 2018b that only assumes inflowing particles would satisfy these constraints). We indicate this schematically in Figure 4, where we show an orthogonal rotator and four different tangents pointing in the observer direction for a particular $\zeta$. Outflowing and inflowing particles with respect to the nearest magnetic pole are indicated by blue and red arrows, respectively. Blue arrows thus occur in the top half of the meridional slice (large magnetic longitude), and red ones in the bottom (small magnetic longitude). Dark and light colors are used to further distinguish between solutions. The meaning of colors remains identical in Figure 5, where we indicate constraints involving the magnetic colatitude $\theta_{\mathrm{B}}$ and longitude $\phi_{\mathrm{B}}$ (both defined with respect to the magnetic dipole moment axis $\boldsymbol{\mu})$. These constraints derive from the condition that the magnetic field tangents are sampled by the observer, for $\alpha=87^{\circ}$ and $\zeta=60^{\circ}$. For $\theta_{\mathrm{B}}$, the solutions satisfy Equation (34) and its reflection given in Wadiasingh et al. (2018).

The different panels in Figure 5 are 2D projections of the 3D plot in the leftmost corner. The top left panel indicates that the "red solutions" are located around $\phi_{\mathrm{B}} \sim 0$ while the blue ones
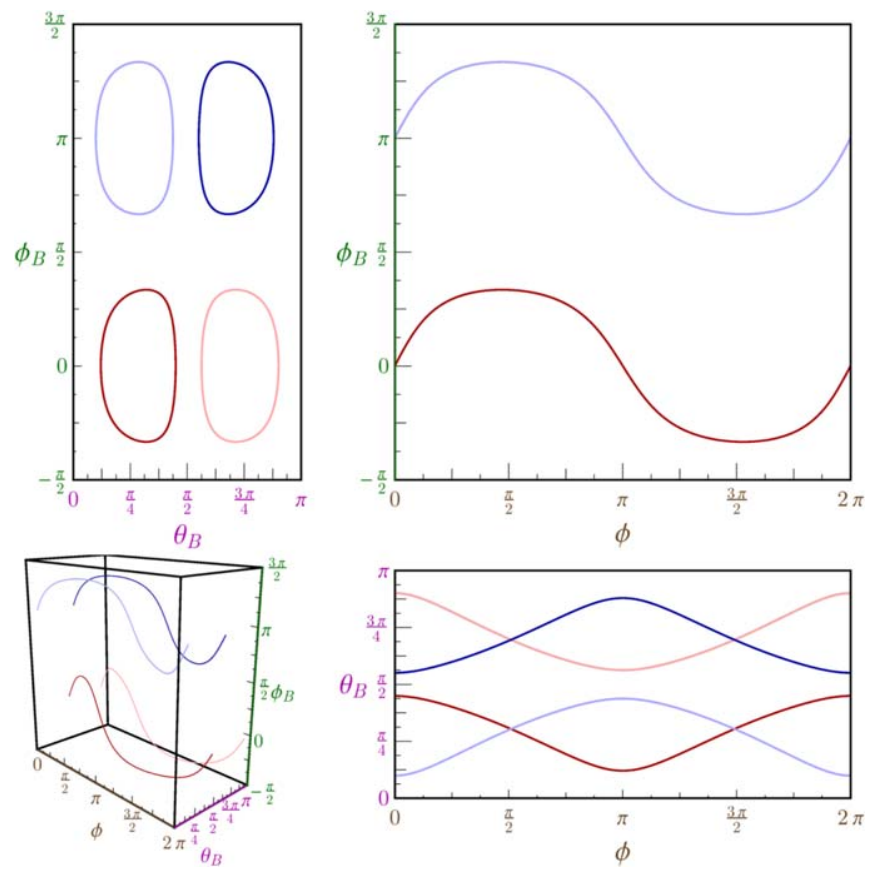

Figure 5. Magnetic colatitudes $\theta_{\mathrm{B}}$ and longitudes $\phi_{\mathrm{B}}$ (with respect to $\boldsymbol{\mu}$ ) which the observer samples during one full rotation in WD spin phase $\phi$ for a static dipole magnetosphere. As in Figure 4, the red and blue colors denote inflowing and outflowing charges in a particular magnetic hemisphere (as defined by $\phi_{\mathrm{B}}$ ), defined with respect to the closest magnetic pole. The magnetic poles are located at $\theta_{\mathrm{B}}=0, \pi$.

occur at $\phi_{\mathrm{B}} \sim \pi$ (i.e., in meridional and antimeriodional planes with respect to $\boldsymbol{\mu})$. In the top right panel, the light and dark colored lines coincide, indicating that the observer samples the same $\phi_{\mathrm{B}}$ for each color during the course of one rotation of the $\mathrm{WD}$, independent of $\theta_{\mathrm{B}}$. The red and blue solutions are of similar functional form, but offset by a factor of $\pi$ in $\phi_{\mathrm{B}}$, as previously. The bottom right projection indicates that the light and dark red solutions have the same form (offset by a factor $\sim \pi / 2$ in $\theta_{\mathrm{B}}$, and the same for the light and dark blue), but the red versus blue ones are mirror images of each other (being $\phi \sim \pi$ out of phase), since they originate close to opposite magnetic poles. Thus, the observer would sample the light blue and dark red solutions (or light red and dark blue ones), offset by half a rotation in spin phase $\phi$, even though they trace out the same range in $\theta_{\mathrm{B}}$.

The constrained spatial region that follows from our RVM fits suggests that a large portion of the magnetosphere, at multiple altitudes, must support relativistic charges. Depending on scenarios of particle acceleration, charges may be either ingoing or outgoing relative to the WD surface, halving the number of potential sites of emission, unless there are counterstreaming beams. In a more complete emission model beyond the geometric RVM, deriving constraints on the altitude of emission should be possible, thereby pinning down the precise location in the magnetosphere where emission arises at any phase.

Interestingly, the estimated polar cap opening angle is much lower $\left(\theta_{\mathrm{PC}} / 2 \pi \sim 1 \%\right)$ than the duty cycle $\sim 100 \%$, implying that the two-star interaction may lead to a much larger opening angle, or that the emission comes from relatively high up, originating on flaring magnetic field lines. 


\subsection{Constraining the Emission Mechanism}

In order to constrain the emission mechanism that might be responsible for the polarized optical radiation, let us estimate several relevant quantities and compare pertinent timescales and length scales. The magnetic field at the polar cap may be estimated assuming $M_{\mathrm{WD}}=1.0 M_{\odot}$ via

$$
\begin{aligned}
B_{\mathrm{p}} & \sim 8 \times 10^{8} \mathrm{G} \\
& \times\left(\frac{R_{\mathrm{WD}}}{7 \times 10^{8} \mathrm{~cm}}\right)^{-3}\left(\frac{P}{117 \mathrm{~s}}\right)^{1 / 2}\left(\frac{\dot{P}}{3.9 \times 10^{-13} \mathrm{~s} \mathrm{~s}^{-1}}\right)^{1 / 2},
\end{aligned}
$$

from dipole spin-down, with $R_{\mathrm{WD}}$ the WD radius. Geng et al. (2016) calculates the magnetic field strength $B_{\mathrm{x}}$ at a distance $x=a-R_{\mathrm{WD}} \sim 5 \times 10^{10} \mathrm{~cm}$ above the stellar surface, where $a$ is binary separation, obtaining $B_{\mathrm{x}}=B_{\mathrm{p}}\left(x / R_{\mathrm{WD}}\right)^{-3} \sim 2 \times$ $10^{3} \mathrm{G}$. We estimate the potential drop at the polar cap using (Goldreich \& Julian 1969)

$$
\begin{aligned}
& \Phi_{\max } \sim 4 \times 10^{11} \text { statvolt } \\
& \times\left(\frac{R_{\mathrm{WD}}}{7 \times 10^{8} \mathrm{~cm}}\right)^{3}\left(\frac{B_{\mathrm{p}}}{8 \times 10^{8} \mathrm{G}}\right)\left(\frac{P}{117 \mathrm{~s}}\right)^{-2} .
\end{aligned}
$$

This yields a maximum Lorentz factor of $\gamma_{\text {max }, \Phi} \sim 3 \times 10^{8}$, although it is unlikely that the particle will be able to tap the full potential.

The corresponding SR, CR, and inverse Compton (IC) timescales $(\gamma / \dot{\gamma})$ are indicated in Figure 6, along with an acceleration $\left(R_{\mathrm{L}} / c\right)$ and crossing timescale $(a / c)$, where $R_{\mathrm{L}}$ is the Larmor radius. To calculate the IC loss rate we used $\dot{E}_{\mathrm{IC}}=$ $4 \sigma_{\mathrm{T}} c U \gamma_{\mathrm{e}}^{2} \gamma_{\mathrm{KN}}^{2} / 3\left(\gamma_{\mathrm{e}}^{2}+\gamma_{\mathrm{KN}}^{2}\right)$ (Schlickeiser \& Ruppel 2010), where $\gamma_{\mathrm{KN}}=3 \sqrt{5} m_{\mathrm{e}} c^{2} / 8 \pi k T$ and $U=2 \sigma_{\mathrm{SB}} T^{4} R_{\star}^{2} / c R_{0}^{2}$ is the photon energy density. Here, $m_{\mathrm{e}}$ is the electron mass, $\sigma_{\mathrm{T}}$ is the Thomson cross section, $\sigma_{\mathrm{SB}}$ is the Stefan-Boltzmann constant, $k$ is the Boltzmann constant, $R_{\star}$ is the companion radius, and $R_{0}$ is the distance from the companion's center to the shock. We consider a "near" $\left(B_{\mathrm{p}}\right)$ and "far" $\left(B_{\mathrm{x}}\right)$ case. Since the IC cooling time is much larger than that of SR (even for small pitch angles), we can rule out IC.

If we equate the SR and CR loss rates to solve for the average pitch angle $\eta$, this yields a maximum value of

$$
\sin \eta \sim 4 \times 10^{-3}\left(\frac{\gamma}{3 \times 10^{8}}\right)\left(\frac{B}{2 \times 10^{3} \mathrm{G}}\right)^{-1},
$$

where $e$ is electron charge and $\rho_{\mathrm{c}} \sim 4 R_{\mathrm{WD}} / 3 \theta_{\mathrm{pc}}$ the curvature radius at the surface of the WD, with $\theta_{\mathrm{PC}} \sim\left(\Omega R_{\mathrm{WD}} / c\right)^{1 / 2}$. Thus, we see that SR dominates for all reasonable values of $\gamma$ and for nonzero pitch angles, with CR only becoming relevant for $\gamma \gtrsim \gamma_{\text {max }, \Phi}$ or for pitch angles $\eta \lesssim 10^{-3}$.

Following Takata et al. (2018) and attributing the nonthermal pulsed X-ray emission to a power-law tail that is emitted by a power-law particle spectrum between $\gamma_{\min }$ and $\gamma_{\max }$, we can infer constraints using the observed frequencies that correspond to the peak in the spectral energy density $\left(\nu_{\mathrm{obs}, \min }=2 \times 10^{13} \mathrm{~Hz}\right)$ and the highest pulsed X-ray photon $\left(\nu_{\mathrm{obs}, \max } \gtrsim 2 \times 10^{18} \mathrm{~Hz}\right)$ :

$$
\gamma_{\min }^{2} B \sin \eta \sim 5 \times 10^{6} \mathrm{G}\left(\frac{\nu_{\mathrm{obs}, \min }}{2 \times 10^{13} \mathrm{~Hz}}\right),
$$

$$
\gamma_{\max }^{2} B \sin \eta \gtrsim 5 \times 10^{11} \mathrm{G}\left(\frac{\nu_{\mathrm{obs}, \max }}{2 \times 10^{18} \mathrm{~Hz}}\right) .
$$

When enforcing that $B<B_{\mathrm{p}}$, we obtain

$$
\begin{gathered}
\gamma_{\min } \gtrsim 30\left(\frac{\nu_{\mathrm{obs}, \min }}{2 \times 10^{13} \mathrm{~Hz}}\right)\left(\frac{\sin \eta}{10^{-5}}\right)^{-1 / 2}, \\
\gamma_{\max } \gtrsim 8 \times 10^{3}\left(\frac{\nu_{\mathrm{obs}, \max }}{2 \times 10^{18} \mathrm{~Hz}}\right)\left(\frac{\sin \eta}{10^{-5}}\right)^{-1 / 2},
\end{gathered}
$$

for a fiducial value of $\sin \eta \sim 10^{-5}$. (Imposing a lower limit of $\gamma_{\text {min }} \approx 5$ formally constrains $\eta \gtrsim 2 \times 10^{-4}$.) These constraints are consistent with the assumptions made by Takata et al. (2017) who model the SR spectrum assuming $\gamma_{\min }=50$ and $\gamma_{\max }=5 \times 10^{6}$.

Let us calculate a characteristic length scale for both SR and CR:

$$
\begin{aligned}
L_{\mathrm{SR}} \approx & c \frac{\gamma}{\dot{\gamma}_{\mathrm{SR}}}=10^{10} \mathrm{~cm} \\
& \times\left(\frac{\nu_{\mathrm{obs}, \mathrm{min}}}{2 \times 10^{13} \mathrm{~Hz}}\right)^{-1 / 2}\left(\frac{\mathrm{B}}{8 \times 10^{8} \mathrm{G}} \frac{\sin \eta}{10^{-5}}\right)^{-3 / 2}
\end{aligned}
$$

and

$$
\begin{aligned}
L_{\mathrm{CR}} & =c \frac{\gamma}{\dot{\gamma}_{\mathrm{CR}}} \\
& =10^{20} \mathrm{~cm}\left(\frac{\nu_{\mathrm{obs}, \mathrm{min}}}{2 \times 10^{13} \mathrm{~Hz}}\right)^{-1}\left(\frac{\rho_{\mathrm{c}}}{5 \times 10^{10} \mathrm{~cm}}\right) .
\end{aligned}
$$

Since $L_{\mathrm{SR}} \ll L_{\mathrm{CR}}$, it is apparent that SR should dominate over $\mathrm{CR}$, even for very small values for $\eta$ (see Figure 6).

Observations by Buckley et al. (2017) indicate that the degree of linear polarization of the optical data varies with orbital phase, but may reach up to $40 \%$. This constraint should be exploited in an emission model, but is beyond the RVM's capabilities. For example, Takata \& Cheng (2019) argue that a polarization degree that varies with orbital phase may be understood in the framework of different contributions of the SR and the thermal emission from the companion, since the latter may depolarize the observed emission. We expect that a model that includes emission from different emission heights that bunch in phase to form the peaks will not likely overpredict the maximum observed polarization degree of $40 \%$. SR from a single particle in an ordered field may reach high values, but this will be lowered by contributions from several particles within the population of radiating particles emitting at different spatial locales. This is similar to the findings of Harding \& Kalapotharakos (2017) who found large PA swings and deep depolarization dips during the light-curve peaks in all energy bands in their multiwavelength pulsar model that invoke caustic emission. Such a model may not be exactly applicable to the WD pulsar, although contributions from emitting particles at different altitudes may provide a blended polarization signature, thus lowering the polarization degree versus that expected from a single particle in an ordered field. The detailed characteristics depend on emission position and mechanism.

A constraint on $B$ can be obtained by assuming that the cyclotron energy is below the SED peak (otherwise a break would be observable due to this threshold energy). At the WD surface, the cyclotron energy is $9 \mathrm{eV}$. When $\nu_{\text {cycl }}=\nu_{\mathrm{obs}, \min }$, $B \lesssim 7 \times 10^{6} \mathrm{G}$, implying that the emission site is at least 
$\sim 5 R_{\mathrm{WD}}$ above the stellar surface. Another constraint on $B$ may be obtained by requiring the ratio of electromagnetic to kinetic particle energy density to be $\sigma \gg 1$ (since the RVM results point to particles following the local field), but this limit is not very constraining due to the uncertain emission volume. On the other hand, the plasma density should be high enough to explain the observed flux of nonthermal emission. To estimate a lower limit for the plasma density (since the charge density may be much lower than the actual number density) we can calculate the Goldreich-Julian number density $n_{\mathrm{GJ}}$ (Goldreich \& Julian 1969):

$$
\begin{aligned}
\left|n_{\mathrm{GJ}}\right| & =\frac{\boldsymbol{\Omega} \cdot \boldsymbol{B}}{2 \pi e c} \\
& =4.7 \times 10^{5} \mathrm{~cm}^{-3}\left(\frac{\mathrm{P}}{117 \mathrm{~s}}\right)^{-1}\left(\frac{\mathrm{B}}{8 \times 10^{8} \mathrm{G}}\right) \\
& =1.2 \mathrm{~cm}^{-3}\left(\frac{\mathrm{P}}{117 \mathrm{~s}}\right)^{-1}\left(\frac{\mathrm{B}}{2 \times 10^{3} \mathrm{G}}\right),
\end{aligned}
$$

with $\Omega$ being the angular frequency. The number of particles needed to explain the SR spectrum may be estimated as follows. Let us focus on the peak frequency $\nu_{\text {peak }}=0.29 \nu_{\mathrm{c}}$, with $\nu_{\mathrm{c}}=$ $3 e \gamma^{2} B \sin \eta / 4 \pi m_{\mathrm{e}} c$. We use $F\left(\nu_{\text {peak }} / \nu_{\mathrm{c}}\right)=F(0.29)=0.924$ and assume a delta distribution for the steady-state particle spectrum $d N / d E_{\mathrm{e}}=N_{0} \delta\left(E_{\mathrm{e}}-E_{\mathrm{e}}^{*}\right)$, with $E_{\mathrm{e}}^{*}=\gamma m_{\mathrm{e}} c^{2}$ the energy needed to explain the peak of the spectrum at $0.29 \nu_{\mathrm{c}}$. From Figure 5 of Takata \& Cheng (2019), we take $\nu F_{\nu}^{\text {obs }}=8 \times$ $10^{-12} \mathrm{erg} \mathrm{s}^{-1} \mathrm{~cm}^{-2}$ at an observed energy of $\sim 0.02 \mathrm{eV}$. Using

$$
P_{\nu}=\frac{\sqrt{3} e^{3} B \sin \eta}{m_{\mathrm{e}} c^{2}} F\left(\nu_{\text {peak }} / \nu_{\mathrm{c}}\right)
$$

and a rough estimate of the emitting volume $V \sim 2 \pi(1-$ $\left.\cos \theta_{\mathrm{PC}}\right) a^{3} / 3 \sim 10^{30} \mathrm{~cm}^{3}$, we find

$$
N_{0} \sim 5 \times 10^{36}
$$

and thus ${ }^{10}$

$$
n_{\mathrm{e}} \sim \frac{N_{0}}{V} \sim 10^{6} \mathrm{~cm}^{-3} .
$$

This is lower than the estimate of Geng et al. (2016) who find $n_{\mathrm{e}} \sim 4 \times 10^{8} \mathrm{~cm}^{-1}$, but still highly supra-Goldreich-Julian suggesting screening of $E$ fields and electron-ion plasma sourced from the companion. On the other hand, since we have demonstrated that the PPA of the WD can be modeled by the RVM, this could mean that the emission of the AR Sco system originates from a dipole-like magnetosphere surrounding the WD (Buckley et al. 2017). Using observations of the linear flux, circular flux, and PPA observations, Potter \& Buckley (2018b) show that the polarization is coupled to the WD spin period, agreeing with our conclusion, while models that place the emission site at the companion fail to explain the polarization signatures that are clearly coupled to the spin period of the WD. The "missing" particles noted by Geng et al. (2016) may either be supplied by the companion that may

\footnotetext{
${ }^{10}$ One may use this rough estimate of particle number density as well as assumptions for/limits on $\sigma=B^{2} /\left(8 \pi n_{\mathrm{e}} \gamma m_{\mathrm{e}} c^{2}\right)$ at different spatial positions implying different values of $B$, to constrain the average $\gamma$ at those positions. For example, at the companion position, one would obtain $\langle\gamma\rangle \lesssim 10^{4}$ for $\sigma>1$.
}

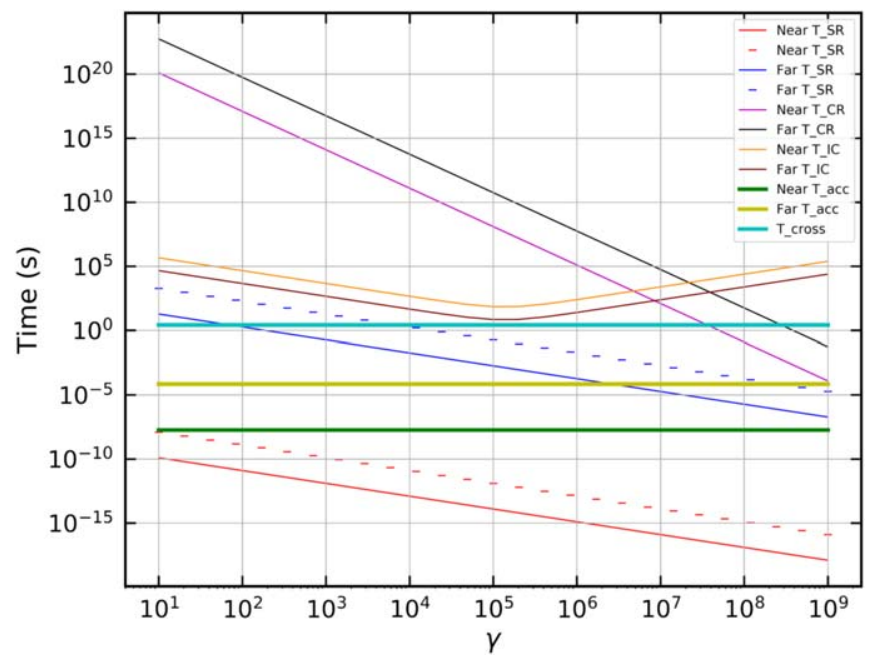

Figure 6. Acceleration, crossing, and radiation (CR, SR, and IC) loss timescales for "far" $\left(B_{\mathrm{x}}\right)$ and "near" $\left(B_{\mathrm{p}}\right)$ cases. The solid lines for SR represent a pitch angle of $\pi / 2$ and the dashed lines represent a pitch angle of $0.1\left(\sim 6^{\circ}\right)$. For this figure, we used a companion temperature of $k T=1 \mathrm{eV}$, i.e., $T \sim 12,000 \mathrm{~K}$ and radius of $R_{\text {comp }}=0.36 R_{\odot}$.

inject relativistic electrons into the WD magnetosphere, or less likely by pair cascades (probably needing severely nonpolar B-field structures) occurring in the WD magnetosphere, and does not per se argue for an emission location near a putative bow shock. Moreover, freshly injected particles from the companion may solve the issue of needing nonzero pitch angles for SR to dominate.

\section{Conclusions}

We applied the RVM to optical polarization data of AR Sco. We obtained $\alpha \sim 90^{\circ}$ confirming the value expected from light-curve inspection and a $180^{\circ}$ PPA swing with spin phase. Finding $\zeta \sim 60^{\circ}$ also agrees with the independent assumption by Potter \& Buckley (2018b) who adopted $\zeta=60^{\circ}$ for their model to reproduce the observed data. Using the result of $\zeta=60^{\circ}$ we then found that $m_{\mathrm{WD}}=1.00_{-0.13}^{+0.19} M_{\odot}$, which is within the limits by Marsh et al. (2016). We next obtained $q=3.45_{-0.45}^{+0.66}$, which is slightly larger compared to the value of $q=2.67$ calculated by Marsh et al. (2016) when adopting $m_{\mathrm{WD}}=0.8$. Our fits of the PPA evolution of the WD using the RVM could imply that the emission of the AR Sco system originates from the dipole-like magnetosphere of the WD, probably close to its polar caps, while the particles are probably being accelerated at and injected from the companion star. The issue of pitch angle evolution should be addressed with detailed emission modeling.

Future work includes fitting the RVM to PPA data in other energy bands, applying a general polarization calculation that includes special-relativistic corrections and investigating the effect of different types of magnetic field structures on the predicted polarization signatures. We will also model the phase-resolved PPA to infer $\alpha$ and $\zeta$ for different orbital phases, which may constrain effects of spin precession in this system. This work highlights the complementary constraints on system geometry, emission locales, and radiation physics that are supplied by adding polarization data to spectral and temporal data within a unified approach. 
We thank the anonymous referee for insightful questions, comments, and suggestions. Z.W. thanks Demos Kazanas for helpful discussions. This work is based on the research supported wholly/in part by the National Research Foundation of South Africa (NRF; grant No. 99072). The Grantholder acknowledges that opinions, findings, and conclusions or recommendations expressed in any publication generated by the NRF supported research is that of the author(s), and that the NRF accepts no liability whatsoever in this regard. Z.W. is thankful for support from the NASA Postdoctoral Program. This work has made use of the NASA Astrophysics Data System.

Software: emcee (Foreman-Mackey et al. 2013).

\section{Appendix \\ RVM Atlas}

In Figure 7, we indicate the behavior of the RVM for different choices of $\alpha$ and $\zeta$.

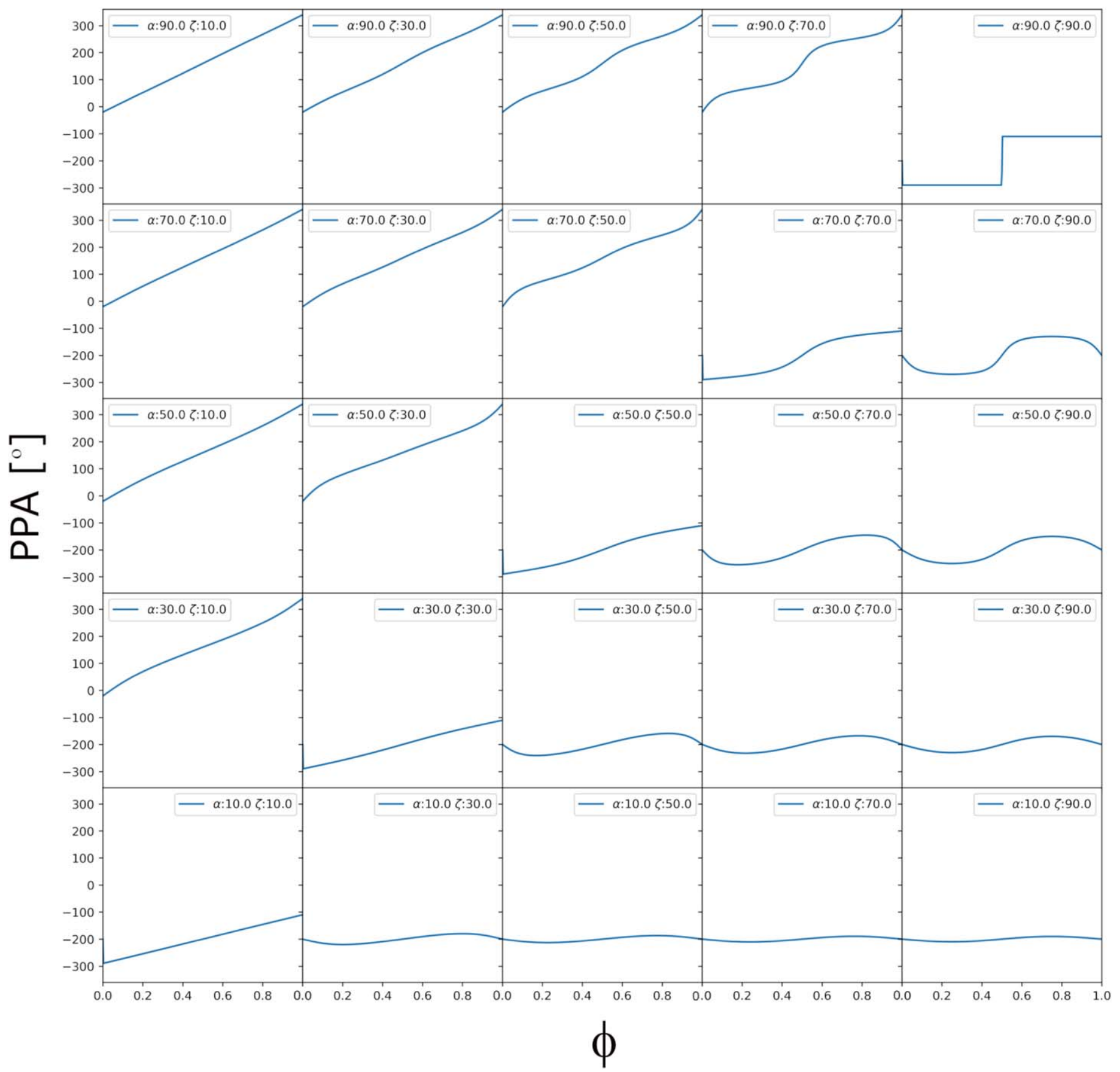

Figure 7. Atlas of RVM for different choices of $\alpha$ and $\zeta$ (in degrees) as indicated in the legend of each subplot. 


\section{ORCID iDs}

Louis du Plessis (1) https://orcid.org/0000-0002-5158-4152

Zorawar Wadiasingh (ib https://orcid.org/0000-00029249-0515

Christo Venter (1) https://orcid.org/0000-0002-2666-4812

Alice K. Harding (i) https://orcid.org/0000-0001-6119-859X

\section{References}

Albrecht, S., Reffert, S., Snellen, I., Quirrenbach, A., \& Mitchell, D. S. 2007, A\&A, 474, 565

Barker, B. M., \& O'Connell, R. F. 1975a, PhRvD, 12, 329

Barker, B. M., \& O'Connell, R. F. 1975b, ApJL, 199, L25

Buckley, D. A. H., Meintjes, P. J., Potter, S. B., Marsh, T. R., \& Gänsicke, B. T. 2017, NatAs, 1, 0029

Cordes, J. M., Wasserman, I., \& Blaskiewicz, M. 1990, ApJ, 349, 546

Damour, T., \& Ruffini, R. 1974, CRASM, 279, 971

Desvignes, G., Kramer, M., Kejia, L., et al. 2019, Sci, 365, 1013

du Plessis, L., Wadiasingh, Z., Venter, C., et al. 2019, arXiv:1907.01311

Esposito, L. W., \& Harrison, E. R. 1975, ApJL, 196, L1

Everett, J. E., \& Weisberg, J. M. 2001, ApJ, 553, 341
Foreman-Mackey, D., Hogg, D. W., Lang, D., \& Goodman, J. 2013, PASP, 125,306

Geng, J.-J., Zhang, B., \& Huang, Y.-F. 2016, ApJL, 831, L10

Goldreich, P., \& Julian, W. H. 1969, ApJ, 157, 869

Harding, A. K., \& Kalapotharakos, C. 2017, ApJ, 840, 73

Harding, A. K., Usov, V. V., \& Muslimov, A. G. 2005, ApJ, 622, 531

Katz, J. I. 2017, ApJ, 835, 150

Kramer, M. 1998, ApJ, 509, 856

Marsh, T. R., Gänsicke, B. T., Hümmerich, S., et al. 2016, Natur, 537, 374

Paczyński, B. 1971, ARA\&A, 9, 183

Peterson, E., Littlefield, C., \& Garnavich, P. 2019, AJ, 158, 131

Potter, S. B., \& Buckley, D. A. H. 2018a, MNRAS, 478, L78

Potter, S. B., \& Buckley, D. A. H. 2018b, MNRAS, 481, 2384

Radhakrishnan, V., \& Cooke, D. J. 1969, ApL, 3, 225

Schlickeiser, R., \& Ruppel, J. 2010, NJPh, 12, 033044

Stiller, R. A., Littlefield, C., Garnavich, P., et al. 2018, AJ, 156, 150

Takata, J., \& Cheng, K. S. 2019, ApJ, 875, 119

Takata, J., Hu, C.-P., Lin, L. C. C., et al. 2018, ApJ, 853, 106

Takata, J., Yang, H., \& Cheng, K. S. 2017, ApJ, 851, 143

Wadiasingh, Z., Baring, M. G., Gonthier, P. L., \& Harding, A. K. 2018, ApJ, 854,98

Watson, C. A., Littlefair, S. P., Diamond, C., et al. 2011, MNRAS, 413, L71

Weisberg, J. M., Romani, R. W., \& Taylor, J. H. 1989, ApJ, 347, 1030 